PROCEEDINGS OF THE

AMERICAN MATHEMATICAL SOCIETY

Volume 135, Number 8, August 2007, Pages 2483-2495

S 0002-9939(07)08710-2

Article electronically published on March 14, 2007

\title{
ON CLARKSON-MCCARTHY INEQUALITIES FOR $n$-TUPLES OF OPERATORS
}

\author{
EDWARD KISSIN
}

(Communicated by N. Tomczak-Jaegermann)

\begin{abstract}
In this paper we obtain analogues of Clarkson-McCarthy inequalities for $n$-tuples of operators from Schatten ideals $C_{p}$. Using them, we extend the results of Bhatia and Kittaneh on inequalities for partitioned operators and for Cartesian decomposition of operators from $C_{p}$.
\end{abstract}

\section{INTRODUCTION}

Noncommutative analogues of Clarkson inequalities for pairs of operators in Schatten ideals obtained by McCarthy in [M] play an important role in analysis and operator theory. They were generalized for general symmetric norms by Bhatia and Holbrook [BH] and Hirzallah and Kittaneh $[\mathrm{HK}$. In BK3. Bhatia and Kittaneh proved analogues of Clarkson-McCarthy inequalities for $n$-tuples of operators of special type. In this paper we obtain analogues of Clarkson-McCarthy inequalities for all $n$-tuples of operators. Using them, we extend the results of Bhatia and Kittaneh [BK1, BK2] on inequalities for partitioned operators and for Cartesian decompositions of operators.

Let $(B(H),\|\cdot\|)$ be the algebra of all bounded operators on a separable Hilbert space $H$ and let $C(H)$ be the ideal of all compact operators in $B(H)$. A two-sided ideal $J$ of $B(H)$ is symmetrically normed (s.n.) if (see [GK]) it is a Banach space in its own norm $\|\cdot\|_{J}$ and

$$
\|A X B\|_{J} \leq\|A\|\|X\|_{J}\|B\| \text { for } A, B \in B(H) \text { and } X \in J .
$$

By Calkin's theorem, all s. n. ideals lie in $C(H)$.

Let $A \in C(H)$ and let $\left\{s_{j}\right\}$ be all eigenvalues of the operator $|A|=\left(A^{*} A\right)^{\frac{1}{2}}$. For $0<p<\infty$, all

$$
C_{p}=\left\{A \in C(H):\|A\|_{p}=\left(\sum_{j} s_{j}^{p}\right)^{1 / p}<\infty\right\}
$$

are linear spaces. For $1 \leq p<\infty$, each $C_{p}$ is a Schatten s.n. ideal of $B(H)$ with norm $\|\cdot\|_{p}$.

Received by the editors November 22, 2005 and, in revised form March 13, 2006.

2000 Mathematics Subject Classification. Primary 47A30; Secondary 46B20, 47B10.

(C)2007 American Mathematical Society Reverts to public domain 28 years from publication 
Set $q=\frac{p}{p-1}$, if $1<p<\infty$. McCarthy [M] (see also [S]) obtained the following noncommutative analogues of Clarkson estimates for $L_{p}$-spaces:

$$
\begin{aligned}
2\left(\|A\|_{p}^{p}+\|B\|_{p}^{p}\right) & \leq\|A+B\|_{p}^{p}+\|A-B\|_{p}^{p} \leq 2^{p-1}\left(\|A\|_{p}^{p}+\|B\|_{p}^{p}\right), \\
2\left(\|A\|_{p}^{p}+\|B\|_{p}^{p}\right)^{q / p} & \leq\|A+B\|_{p}^{q}+\|A-B\|_{p}^{q},
\end{aligned}
$$

for $A, B \in C_{p}$, if $2 \leq p<\infty$. For $1<p<2$ the above inequalities are reversed.

Hirzallah and Kittaneh $\mathrm{HK}$ generalized (1) and proved that, for any s.n. ideal $J$ and $A, B \in J$,

$$
2\left(\left\||A|^{p}+|B|^{p}\right\|_{J}\right) \leq\left\||A+B|^{p}+|A-B|^{p}\right\|_{J} \leq 2^{p-1}\left(\left\||A|^{p}+|B|^{p}\right\|_{J}\right),
$$

if $2 \leq p<\infty$. For $0<p<2$, these inequalities are reversed.

Denote by $C_{p}(n)$ the linear space of all columns $\bar{A}=\left(A_{j}\right), 1 \leq j \leq n$, with $A_{j} \in C_{p}$. Let $H^{n}$ be the orthogonal sum of $n$ copies of the Hilbert space $H$. Each operator $R$ in $B\left(H^{n}\right)$ can be represented as an $n \times n$ block-matrix operator $R=\left(R_{j k}\right)$ with $R_{j k} \in B(H)$. Bhatia and Kittaneh in [BK3] obtained analogues of inequalities (11)-(3) for $n$-tuples of operators $\bar{A} \in C_{p}(n)$. Let $\bar{B}=R \bar{A}$, where the $R_{j k}$ have the particular form

$$
R_{j k}=\exp \left(i \frac{2 \pi(j-1)(k-1)}{n}\right) \mathbf{1}
$$

They proved that if $2 \leq p<\infty$, then

$$
\begin{gathered}
n^{\frac{2}{p}} \sum_{j=1}^{n}\left\|A_{j}\right\|_{p}^{2} \leq \sum_{j=1}^{n}\left\|B_{j}\right\|_{p}^{2} \leq n^{2-\frac{2}{p}} \sum_{j=1}^{n}\left\|A_{j}\right\|_{p}^{2}, \\
n \sum_{j=1}^{n}\left\|A_{j}\right\|_{p}^{p} \leq \sum_{j=1}^{n}\left\|B_{j}\right\|_{p}^{p} \leq n^{p-1} \sum_{j=1}^{n}\left\|A_{j}\right\|_{p}^{p}, \\
n\left(\sum_{j=1}^{n}\left\|A_{j}\right\|_{p}^{p}\right)^{q / p} \leq \sum_{j=1}^{n}\left\|B_{j}\right\|_{p}^{q}, \text { where } q=\frac{p}{p-1} .
\end{gathered}
$$

For $0<p<2$, inequalities (5) and (6) are reversed; for $1<p<2$, inequality (7) is reversed.

For a s.n. ideal $J$, denote by $J(n)$ the linear space of all columns $\bar{A}=\left(A_{j}\right)$, $1 \leq j \leq n$, with all $A_{j} \in J$, and set $\bar{B}=R \bar{A}$. It was proved in BK3 that if the $R_{j k}$ have the form given in (4), then

$$
n\left\|\sum_{j=1}^{n}\left|A_{j}\right|^{p}\right\|_{J} \leq\left\|\sum_{j=1}^{n}\left|B_{j}\right|^{p}\right\|_{J} \leq n^{p-1}\left\|\sum_{j=1}^{n}\left|A_{j}\right|^{p}\right\|_{J}, \text { for } 2 \leq p<\infty .
$$

For $0<p<2$, the above inequalities are reversed.

For $n=2$, inequalities (5)-(8) give inequalities (10)-(3).

In [BK3] Bhatia and Kittaneh further extended inequalities (5)-(8) to the case when

$$
R_{j k}=r_{j k} \mathbf{1} \text { with } r_{j k} \in \mathbb{C}, R^{*} R=|z| \mathbf{1}_{n} \text { and } R^{2}=z P,
$$


where $z \in \mathbb{C}$ and $P$ is a permutation matrix. For $\bar{A} \in C_{p}(n)$ and $\bar{B}=R \bar{A}$, they proved that

$$
n^{\frac{\lambda}{p}-\frac{\lambda}{2}}|z|^{\lambda / 2} \sum_{j=1}^{n}\left\|A_{j}\right\|_{p}^{\lambda} \leq \sum_{j=1}^{n}\left\|B_{j}\right\|_{p}^{\lambda} \leq n^{-\left(\frac{\lambda}{p}-\frac{\lambda}{2}\right)}|z|^{\lambda / 2} \sum_{j=1}^{n}\left\|A_{j}\right\|_{p}^{p},
$$

if $2 \leq \lambda \leq p<\infty$,

$$
\sum_{j=1}^{n}\left\|B_{j}\right\|_{p}^{q} \leq r^{q-2}|z|\left(\sum_{j=1}^{n}\left\|A_{j}\right\|_{p}^{p}\right)^{q / p}, \text { if } 1<p \leq 2,
$$

where $r=\max \left|r_{j k}\right|$. For $\bar{A} \in J(n)$ and $\bar{B}=R \bar{A}$, they showed that, for $2 \leq p<\infty$,

$$
n^{1-\frac{p}{2}}|z|^{p / 2}\left\|\sum_{j=1}^{n}\left|A_{j}\right|^{p}\right\|_{J} \leq\left\|\sum_{j=1}^{n}\left|B_{j}\right|^{p}\right\|_{J} \leq n^{-\left(1-\frac{p}{2}\right)}|z|^{p / 2}\left\|\sum_{j=1}^{n}\left|A_{j}\right|^{p}\right\|_{J} .
$$

In this paper we obtain the analogues of inequalities (5)-(12) for all invertible operators $R$ in $B\left(H^{n}\right)$. Denote $\alpha=\left\|R^{-1}\right\|, \beta=\|R\|$. We will prove the following theorems.

Theorem 1. Let $\bar{A} \in C_{p}(n)$ and $\bar{B}=R \bar{A}$, where $R$ is invertible. If $2 \leq p$ and $\lambda, \mu \in[2, p]$, or if $0<p \leq 2$ and $\lambda, \mu \in[p, 2]$, then

$$
n^{-\left|\frac{1}{p}-\frac{1}{2}\right|} \alpha^{-1}\left(\frac{1}{n} \sum_{j=1}^{n}\left\|A_{j}\right\|_{p}^{\mu}\right)^{\frac{1}{\mu}} \leq\left(\frac{1}{n} \sum_{j=1}^{n}\left\|B_{j}\right\|_{p}^{\lambda}\right)^{\frac{1}{\lambda}} \leq n^{\left|\frac{1}{p}-\frac{1}{2}\right|_{\beta}}\left(\frac{1}{n} \sum_{j=1}^{n}\left\|A_{j}\right\|_{p}^{\mu}\right)^{\frac{1}{\mu}} .
$$

Theorem 2. Let $\bar{A} \in C_{p}(n)$ and $\bar{B}=R \bar{A}$ where $R=\left(R_{j k}\right)$ is invertible. Let $r=\max \left\|R_{j k}\right\|, \rho=\max \left\|\left(R^{-1}\right)_{j k}\right\|$ and $q=\frac{p}{p-1}$. Then

$$
\begin{gathered}
\left(\sum_{j=1}^{n}\left\|A_{j}\right\|_{p}^{p}\right)^{\frac{1}{p}} \leq \rho^{1-\frac{2}{p}} \alpha^{\frac{2}{p}}\left(\sum_{j=1}^{n}\left\|B_{j}\right\|_{p}^{q}\right)^{\frac{1}{q}} \text { for } 2 \leq p ; \\
\left(\sum_{j=1}^{n}\left\|B_{j}\right\|_{p}^{q}\right)^{\frac{1}{q}} \leq r^{\frac{2}{p}-1} \beta^{\frac{2}{q}}\left(\sum_{j=1}^{n}\left\|A_{j}\right\|_{p}^{p}\right)^{\frac{1}{p}} \quad \text { for } 1<p \leq 2 .
\end{gathered}
$$

Theorem 3. Let $J$ be an s.n. ideal. For $\bar{A} \in J(n)$, let $\bar{B}=R \bar{A}$, where $R$ is invertible. Then

$$
n^{-\left|\frac{p}{2}-1\right|} \alpha^{-p}\left\|\sum_{j=1}^{n}\left|A_{j}\right|^{p}\right\|_{J} \leq\left\|\sum_{j=1}^{n}\left|B_{j}\right|^{p}\right\|_{J} \leq n^{\left|\frac{p}{2}-1\right|} \beta^{p}\left\|\sum_{j=1}^{n}\left|A_{j}\right|^{p}\right\|_{J} \text { for } 0<p<\infty .
$$

We will prove Theorems 1 and 3 by obtaining some inequalities for the form

$$
(\bar{A}, \bar{A})=A_{1}^{*} A_{1}+\cdots+A_{n}^{*} A_{n} \text { with } \bar{A} \in J(n) .
$$

Denote by $\mathcal{P}_{n}$ the set of all $n$-tuples $P=\left\{P_{j}\right\}_{j=1}^{n}$ of mutually orthogonal infinitedimensional projections in $B(H)$ with $\sum_{j=1}^{n} P_{j}=1$. We will use the inequalities for $(\bar{A}, \bar{A})$ to prove the following result for partitioned operators. 
Theorem 4. Let $\left\{P_{i}\right\}_{i=1}^{n} \in \mathcal{P}_{n},\left\{Q_{j}\right\}_{j=1}^{m} \in \mathcal{P}_{m}$ and let $A \in C_{p}$. For $2 \leq \lambda \leq p<$ $\infty$

$$
(n m)^{\frac{\lambda}{p}-1} \sum_{i, j}\left\|P_{i} A Q_{j}\right\|_{p}^{\lambda} \leq\|A\|_{p}^{\lambda} \leq(n m)^{\frac{\lambda}{2}-1} \sum_{i, j}\left\|P_{i} A Q_{j}\right\|_{p}^{\lambda} .
$$

For $0<p \leq \lambda \leq 2$, the inequalities are reversed.

Theorem 4 extends the results of BK1] proved there for $n=m, P_{i}=Q_{i}, \lambda=2$ and $\lambda=p$.

Let $\bar{A}=\left(A_{j}\right) \in C_{p}(n)$ and let $A_{j}=X_{j}+i Y_{j}$ be their "Cartesion decompositions," where $X_{j}, Y_{j}$ are selfadjoint operators. Using Theorem 11, we will prove the following theorem.

Theorem 5. (i) Let $2 \leq p$. Then, for $0<\lambda \leq p$ and $2 \leq \mu$,

$$
n^{\frac{1}{p}} 2^{\frac{1}{p}-\frac{1}{2}}\left(\frac{1}{n} \sum_{j=1}^{n}\left\|A_{j}\right\|_{p}^{\lambda}\right)^{\frac{1}{\lambda}} \leq\left\|\sum_{j=1}^{n}\left(X_{j}^{2}+Y_{j}^{2}\right)\right\|_{p / 2}^{1 / 2} \leq n^{\frac{1}{2}}\left(\frac{1}{n} \sum_{j=1}^{n}\left\|A_{j}\right\|_{p}^{\mu}\right)^{\frac{1}{\mu}} .
$$

If $0<p \leq 2$, then, for $p \leq \lambda$ and $0<\mu \leq 2$, the inequalities are reversed.

(ii) Set $\tau(p)=\left|\frac{1}{p}-\frac{1}{2}\right|$. If $p \geq 2$ and $\lambda, \mu \in[2, p]$, or if $0<p<2$ and $\lambda, \mu \in[p, 2]$, then

$$
\begin{aligned}
& (2 n)^{-\tau(p)}\left(\frac{1}{n} \sum_{j=1}^{n}\left(\left\|X_{j}\right\|_{p}^{\mu}+\left\|Y_{j}\right\|_{p}^{\mu}\right)\right)^{\frac{1}{\mu}} \leq 2^{\frac{1}{\mu}-\frac{1}{2}}\left(\frac{1}{n} \sum_{j=1}^{n}\left\|A_{j}\right\|_{p}^{\lambda}\right)^{\frac{1}{\lambda}} \\
& \leq(2 n)^{\tau(p)}\left(\frac{1}{n} \sum_{j=1}^{n}\left(\left\|X_{j}\right\|_{p}^{\mu}+\left\|Y_{j}\right\|_{p}^{\mu}\right)\right)^{\frac{1}{\mu}} .
\end{aligned}
$$

For $n=1$, part $(i)$ of Theorem 5 was proved in [BK2, Theorem 1]; part (ii) was proved, for $\mu=2$, in [BK2, Corollary 1].

\section{Proofs of Theorems 1, 4] and [5]}

It follows from the definition of the norm $\|\cdot\|_{p}$ that

$$
\|A\|_{p}^{2}=\left\|A^{*} A\right\|_{\frac{p}{2}}=\left\|A A^{*}\right\|_{\frac{p}{2}}=\left\|A^{*}\right\|_{p}^{2}, \text { for } A \in C_{p} \text { and } 0<p<\infty .
$$

Let $\left\{A_{j}\right\}_{j=1}^{n}$ be positive operators in $C_{p}$. McCarthy in [M] (see also [S] and [BK1]) proved that

$$
\left\|A_{1}\right\|_{p}^{p}+\cdots+\left\|A_{n}\right\|_{p}^{p} \leq\left\|A_{1}+\cdots+A_{n}\right\|_{p}^{p} \text { for } 1 \leq p<\infty .
$$

Bhatia and Kittaneh showed in Lemma 1 and in formula (7) of [BK2] that

$$
\left(\sum_{j=1}^{n}\left\|A_{j}\right\|_{p}^{p}\right)^{\frac{1}{p}} \geq\left\|\sum_{j=1}^{n} A_{j}\right\|_{p} \geq \sum_{j=1}^{n}\left\|A_{j}\right\|_{p} \text { for } 0<p<1 .
$$

The function $f(x)=x^{\lambda}$ is concave on $[0, \infty)$ for $0 \leq \lambda \leq 1$, and convex for $1 \leq \lambda<\infty$. Hence

$$
\begin{gathered}
n^{\lambda-1}\left(x_{1}^{\lambda}+\cdots+x_{n}^{\lambda}\right) \leq\left(x_{1}+\cdots+x_{n}\right)^{\lambda} \text { for } 0 \leq \lambda \leq 1, \\
n^{\lambda-1}\left(x_{1}^{\lambda}+\cdots+x_{n}^{\lambda}\right) \geq\left(x_{1}+\cdots+x_{n}\right)^{\lambda} \text { for } 1 \leq \lambda<\infty,
\end{gathered}
$$


when $x_{i} \geq 0$. For $\bar{A} \in C_{p}(n)$, the operator $(\bar{A}, \bar{A})=A_{1}^{*} A_{1}+\cdots+A_{n}^{*} A_{n}$ belongs to $C_{p / 2}$.

Lemma 6. (i) Let $2 \leq p$ and $\bar{A} \in C_{p}(n)$. For $0<\lambda \leq p$ and $2 \leq \mu$,

$$
n^{\frac{1}{p}}\left(\frac{1}{n} \sum_{j=1}^{n}\left\|A_{j}\right\|_{p}^{\lambda}\right)^{\frac{1}{\lambda}} \leq\left(\sum_{j=1}^{n}\left\|A_{j}\right\|_{p}^{p}\right)^{\frac{1}{p}} \leq\left\|\sum_{j=1}^{n} A_{j}^{*} A_{j}\right\|_{p / 2}^{1 / 2} \leq n^{\frac{1}{2}}\left(\frac{1}{n} \sum_{j=1}^{n}\left\|A_{j}\right\|_{p}^{\mu}\right)^{\frac{1}{\mu}} .
$$

(ii) If $0<p \leq 2$, then, for $p \leq \lambda$ and $0<\mu \leq 2$, all inequalities are reversed.

Proof. Let $2 \leq p$ and let $0<\lambda \leq p$ and $2 \leq \mu$. Making use of the triangle inequality $(t i)$, we prove part $(i)$ :

$$
\begin{gathered}
n^{\frac{1}{p}}\left(\frac{1}{n} \sum_{j=1}^{n}\left\|A_{j}\right\|_{p}^{\lambda}\right)^{\frac{1}{\lambda}}=\left(n^{\frac{\lambda}{p}-1} \sum_{j=1}^{n}\left(\left\|A_{j}\right\|_{p}^{p}\right)^{\lambda / p}\right)^{\frac{1}{\lambda}} \stackrel{\sqrt[17]{\leq}}{\leq}\left(\sum_{j=1}^{n}\left\|A_{j}\right\|_{p}^{p}\right)^{\frac{1}{p}} \\
\stackrel{\underline{14}}{=}\left(\sum_{j=1}^{n}\left\|A_{j}^{*} A_{j}\right\|_{p / 2}^{p / 2}\right)^{\frac{1}{p}} \stackrel{15}{\leq}\left(\left\|\sum_{j=1}^{n} A_{j}^{*} A_{j}\right\|_{p / 2}^{p / 2}\right)^{\frac{1}{p}}=\left\|\sum_{j=1}^{n} A_{j}^{*} A_{j}\right\|_{p / 2}^{1 / 2} \\
\stackrel{(t i)}{\leq}\left(\left(\sum_{j=1}^{n}\left\|A_{j}^{*} A_{j}\right\|_{\frac{p}{2}}\right)^{\frac{\mu}{2}}\right)_{\stackrel{\frac{1}{\mu}}{\frac{118}{\leq}}\left(n^{\frac{\mu}{2}-1} \sum_{j=1}^{n}\left\|A_{j}^{*} A_{j}\right\|_{p / 2}^{\mu / 2}\right)^{\frac{1}{\mu}}}^{\stackrel{144}{=} n^{\frac{1}{2}}\left(\frac{1}{n} \sum_{j=1}^{n}\left\|A_{j}\right\|_{p}^{\mu}\right)^{\frac{1}{\mu}} .}
\end{gathered}
$$

One proves $(i i)$, by replacing $\leq$ by $\geq$, (15) by (16), (ti) by (16) and exchanging (17) and (18).

Let $A_{j} \in C_{p}$ have mutually orthogonal ranges: $A_{k}^{*} A_{j}=0$ if $j \neq k$. Then

$$
\begin{aligned}
\left\|A_{1}^{*} A_{1}+\cdots+A_{n}^{*} A_{n}\right\|_{p / 2} & =\left\|\left(A_{1}+\cdots+A_{n}\right)^{*}\left(A_{1}+\cdots+A_{n}\right)\right\|_{p / 2} \\
& \stackrel{140}{=}\left\|A_{1}+\cdots+A_{n}\right\|_{p}^{2}
\end{aligned}
$$

and Lemma 6 gives us the following estimates for $\left\|\sum_{j=1}^{n} A_{j}\right\|_{p}$, when $2 \leq \mu=\lambda \leq p$ : (19) $n^{\frac{\lambda}{p}-1}\left(\left\|A_{1}\right\|_{p}^{\lambda}+\cdots+\left\|A_{n}\right\|_{p}^{\lambda}\right) \leq\left\|A_{1}+\cdots+A_{n}\right\|_{p}^{\lambda} \leq n^{\frac{\lambda}{2}-1}\left(\left\|A_{1}\right\|_{p}^{\lambda}+\cdots+\left\|A_{n}\right\|_{p}^{\lambda}\right)$.

For $0<p \leq \mu=\lambda \leq 2$, the inequalities are reversed.

Proof of Theorem 4. Let $2 \leq \lambda \leq p$. Set $A_{i}=P_{i} A$. Then $A=\sum_{i} A_{i}$ and, by (19),

$$
n^{\frac{\lambda}{p}-1}\left(\left\|A_{1}\right\|_{p}^{\lambda}+\cdots+\left\|A_{n}\right\|_{p}^{\lambda}\right) \leq\|A\|_{p}^{\lambda} \leq n^{\frac{\lambda}{2}-1}\left(\left\|A_{1}\right\|_{p}^{\lambda}+\cdots+\left\|A_{n}\right\|_{p}^{\lambda}\right) .
$$

We have $A_{i}^{*}=\sum_{j} Q_{j} A_{i}^{*}$. Since all $Q_{j} A_{i}^{*}$ have mutually orthogonal ranges, we have from (19),

$$
m^{\frac{\lambda}{p}-1} \sum_{j=1}^{m}\left\|Q_{j} A_{i}^{*}\right\|_{p}^{\lambda} \leq\left\|A_{i}^{*}\right\|_{p}^{\lambda} \leq m^{\frac{\lambda}{2}-1} \sum_{j=1}^{m}\left\|Q_{j} A_{i}^{*}\right\|_{p}^{\lambda} .
$$

Since $\left\|A_{i}\right\|_{p}=\left\|A_{i}^{*}\right\|_{p}$ and $\left\|Q_{j} A_{i}^{*}\right\|_{p}=\left\|P_{i} A Q_{j}\right\|_{p}$, we obtain (13) by combining the above inequalities. 
For $0<p \leq \lambda \leq 2$, the inequalities are reversed. Theorem 4 is proved.

Denote by $\langle$,$\rangle the scalar product on H$. Let $S, T$ be positive compact operators on $H$ and let $f$ and $g$ be increasing functions on $[0, \infty)$. If $g(\langle S x, x\rangle) \leq f(\langle T x, x\rangle)$, for all $x \in H$ with $\|x\|=1$, then for each s.n. ideal $J$,

$$
f(T) \in J \text { implies } g(S) \in J \text { and }\|g(S)\|_{J} \leq\|f(T)\|_{J} .
$$

Indeed, it follows from the Minimax principal (see [GK, II.1] or [B, p. 58]) that $s_{j}(g(S)) \leq s_{j}(f(T))$ for all $j$. Hence, by [GK, Lemma III.3.2], $g(S) \in J$ and $\|g(S)\|_{J} \leq\|f(T)\|_{J}$. In particular,

$$
0<S \leq T \text { and } T \in J \text { imply } S \in J \text { and }\|S\|_{J} \leq\|T\|_{J} .
$$

Corollary 7. Let $R \in B\left(H^{n}\right)$ and $\beta=\|R\|$. For $\bar{A} \in C_{p}(n)$, set $\bar{B}=R \bar{A}$. Let either 1) $2 \leq p, 0<\lambda \leq p$ and $2 \leq \mu$; or 2) $0<p \leq 2,0<\lambda \leq 2$ and $p \leq \mu$.

Then

$$
\left(\frac{1}{n}\left(\left\|B_{1}\right\|_{p}^{\lambda}+\cdots+\left\|B_{n}\right\|_{p}^{\lambda}\right)\right)^{\frac{1}{\lambda}} \leq \beta n^{\left|\frac{1}{p}-\frac{1}{2}\right|}\left(\frac{1}{n}\left(\left\|A_{1}\right\|_{p}^{\mu}+\cdots+\left\|A_{n}\right\|_{p}^{\mu}\right)\right)^{\frac{1}{\mu}} .
$$

Proof. Since $K=\beta^{2} \mathbf{1}-R^{*} R$ is a positive operator, we have

(22) $\beta^{2}(\bar{A}, \bar{A})=\left(\bar{A}, R^{*} R \bar{A}\right)+(\bar{A}, K \bar{A})=(R \bar{A}, R \bar{A})+\left(K^{1 / 2} \bar{A}, K^{1 / 2} \bar{A}\right) \geq(\bar{B}, \bar{B})$.

Let $2 \leq p, 0<\lambda \leq p$ and $2 \leq \mu$. It follows from Lemma $6(i)$ and (21) that

$$
n^{\frac{1}{p}}\left(\frac{1}{n} \sum_{j=1}^{n}\left\|B_{j}\right\|_{p}^{\lambda}\right)^{\frac{1}{\lambda}} \leq\|(\bar{B}, \bar{B})\|_{p / 2}^{1 / 2} \leq \beta\|(\bar{A}, \bar{A})\|_{p / 2}^{1 / 2} \leq \beta n^{\frac{1}{2}}\left(\frac{1}{n} \sum_{j=1}^{n}\left\|A_{j}\right\|_{p}^{\mu}\right)^{\frac{1}{\mu}} .
$$

Let $0<p \leq 2,0<\lambda \leq 2$ and $p \leq \mu$. It follows from Lemma 6 (ii) and (16) that

$$
n^{\frac{1}{2}}\left(\frac{1}{n} \sum_{j=1}^{n}\left\|B_{j}\right\|_{p}^{\lambda}\right)^{\frac{1}{\lambda}} \leq\|(\bar{B}, \bar{B})\|_{p / 2}^{1 / 2} \leq \beta\|(\bar{A}, \bar{A})\|_{p / 2}^{1 / 2} \leq \beta n^{\frac{1}{p}}\left(\frac{1}{n} \sum_{j=1}^{n}\left\|A_{j}\right\|_{p}^{\mu}\right)^{\frac{1}{\mu}}
$$

which completes the proof.

Proof of Theorem 1. Let $2 \leq p$ and $\lambda, \mu \in[2, p]$. By Corollary 17.

$$
n^{\frac{1}{p}}\left(\frac{1}{n}\left(\left\|B_{1}\right\|_{p}^{\lambda}+\cdots+\left\|B_{n}\right\|_{p}^{\lambda}\right)\right)^{\frac{1}{\lambda}} \leq \beta n^{\frac{1}{2}}\left(\frac{1}{n}\left(\left\|A_{1}\right\|_{p}^{\mu}+\cdots+\left\|A_{n}\right\|_{p}^{\mu}\right)\right)^{\frac{1}{\mu}} .
$$

Since $\bar{A}=R^{-1} \bar{B}$, interchanging in the above inequality $\lambda$ and $\mu, A_{j}$ and $B_{j}$, and replacing $\beta$ by $\alpha$, we have

$$
n^{\frac{1}{p}}\left(\frac{1}{n}\left(\left\|A_{1}\right\|_{p}^{\mu}+\cdots+\left\|A_{n}\right\|_{p}^{\mu}\right)\right)^{\frac{1}{\mu}} \leq \alpha n^{\frac{1}{2}}\left(\frac{1}{n}\left(\left\|B_{1}\right\|_{p}^{\lambda}+\cdots+\left\|B_{n}\right\|_{p}^{\lambda}\right)\right)^{\frac{1}{\lambda}} .
$$

Combining the above inequalities, we complete the proof of this case. The case when $0<p \leq 2$ and $\lambda, \mu \in[p, 2]$ is proved in the same way.

Remarks. 1) Let $R=\left(R_{j k}\right)$ with $R_{j k}$ satisfying (44). Then $\beta=\alpha^{-1}=n^{\frac{1}{2}}$.

(i) Let $\lambda=\mu$ be 2 or $p$. If $2 \leq p$, then Theorem 1 gives formula (5) and, if $0<p<2$, its reverse. 
(ii) Let $2 \leq p=\mu$ in Corollary 7 . For $0<\lambda \leq p$, we have an inequality complementary to (7):

$$
n^{\frac{1}{p}}\left(\frac{1}{n}\left(\left\|B_{1}\right\|_{p}^{\lambda}+\cdots+\left\|B_{n}\right\|_{p}^{\lambda}\right)\right)^{\frac{1}{\lambda}} \leq n\left(\frac{1}{n}\left(\left\|A_{1}\right\|_{p}^{p}+\cdots+\left\|A_{n}\right\|_{p}^{p}\right)\right)^{\frac{1}{p}} .
$$

2) If $R$ satisfies (9), then $\beta=\alpha^{-1}=|z|^{1 / 2}$. For $\lambda=\mu$, Theorem 1 gives formula (10).

For $1 \leq \mu=p<2$, Corollary 7 gives a formula complementary to (11):

$$
n^{\frac{1}{2}}|z|^{\frac{1}{2}}\left(\frac{1}{n}\left(\left\|A_{1}\right\|_{p}^{p}+\cdots+\left\|A_{n}\right\|_{p}^{p}\right)\right)^{\frac{1}{p}} \leq n^{1 / p}\left(\frac{1}{n}\left(\left\|B_{1}\right\|_{p}^{\lambda}+\cdots+\left\|B_{n}\right\|_{p}^{\lambda}\right)\right)^{\frac{1}{\lambda}} .
$$

3) Let $R$ be a unitary operator. If either $2 \leq p$ and $\lambda, \mu \in[2, p]$, or $0<p \leq 2$ and $\lambda, \mu \in[p, 2]$, then Theorem 1 gives

$$
n^{-\left|\frac{1}{p}-\frac{1}{2}\right|}\left(\frac{1}{n} \sum_{j=1}^{n}\left\|A_{j}\right\|_{p}^{\mu}\right)^{\frac{1}{\mu}} \leq\left(\frac{1}{n} \sum_{j=1}^{n}\left\|B_{j}\right\|_{p}^{\lambda}\right)^{\frac{1}{\lambda}} \leq n^{\left|\frac{1}{p}-\frac{1}{2}\right|}\left(\frac{1}{n} \sum_{j=1}^{n}\left\|A_{j}\right\|_{p}^{\mu}\right)^{\frac{1}{\mu}} .
$$

Let $\left\{P_{i}\right\}_{i=1}^{n} \in \mathcal{P}_{n}$ be a set of mutually orthogonal projections and $\sum_{i} P_{i}=\mathbf{1}$. The operator $R=\left(R_{j k}\right)$, with $R_{j k}=P_{(k+j-1) \bmod (n)}$, is unitary. In particular, for $n=2$, for $\mu=\lambda$ between 2 and $p$ and for any projection $P$, we have

$$
\begin{aligned}
2^{-\lambda\left|\frac{1}{p}-\frac{1}{2}\right|}\left(\left\|A_{1}\right\|_{p}^{\lambda}+\left\|A_{2}\right\|_{p}^{\lambda}\right) & \leq\left\|P A_{1}+(\mathbf{1}-P) A_{2}\right\|_{p}^{\lambda}+\left\|(\mathbf{1}-P) A_{1}+P A_{2}\right\|_{p}^{\lambda} \\
& \leq 2^{\lambda\left|\frac{1}{p}-\frac{1}{2}\right|}\left(\left\|A_{1}\right\|_{p}^{\lambda}+\left\|A_{2}\right\|_{p}^{\lambda}\right) .
\end{aligned}
$$

4) Let $\left\{a_{i}\right\}_{i=1}^{n}$ be positive numbers. The function $g(\lambda)=\left(\frac{1}{n} \sum_{j=1}^{n} a_{j}^{\lambda}\right)^{\frac{1}{\lambda}}$ is monotone increasing. Therefore we get the sharpest inequalities in Theorem 1 if, for $0<p \leq 2$, we set $\lambda=2$ and $\mu=p$; and if, for $2 \leq p<\infty$, we set $\lambda=p$ and $\mu=2$ :

$$
\begin{aligned}
\left\|B_{1}\right\|_{p}^{2}+\cdots+\left\|B_{n}\right\|_{p}^{2} & \leq \beta^{2}\left(\left\|A_{1}\right\|_{p}^{p}+\cdots+\left\|A_{n}\right\|_{p}^{p}\right)^{\frac{2}{p}}, \text { for } 0<p \leq 2, \\
\left(\left\|B_{1}\right\|_{p}^{p}+\cdots+\left\|B_{n}\right\|_{p}^{p}\right)^{\frac{2}{p}} & \leq \beta^{2}\left(\left\|A_{1}\right\|_{p}^{2}+\cdots+\left\|A_{n}\right\|_{p}^{2}\right), \text { for } 2 \leq p<\infty .
\end{aligned}
$$

Let $n=2$ and $R=\left(r_{i j} \mathbf{1}\right), r_{11}=r_{12}=r_{21}=-r_{22}=1$. Then $\beta=\alpha^{-1}=2^{1 / 2}$ and we have

$$
\left\|A_{1}\right\|_{p}^{2}+\left\|A_{2}\right\|_{p}^{2} \leq \frac{1}{2}\left(\left\|A_{1}+A_{2}\right\|_{p}^{p}+\left\|A_{1}-A_{2}\right\|_{p}^{p}\right)^{\frac{2}{p}}, \text { if } 0<p \leq 2 .
$$

For $2 \leq p<\infty$, the inequality is reversed.

Compare (23) to the following inequalities proved by Ball, Carlen and Lieb in BCL:

$$
\left\|A_{1}\right\|_{p}^{2}+(p-1)\left\|A_{2}\right\|_{p}^{2} \leq 2^{-\frac{2}{p}}\left(\left\|A_{1}+A_{2}\right\|_{p}^{p}+\left\|A_{1}-A_{2}\right\|_{p}^{p}\right)^{\frac{2}{p}}, \text { for } 1 \leq p \leq 2,
$$

and the reverse for $2 \leq p<\infty$. If we symmetrize (24), by exchanging $A_{1}$ and $A_{2}$ and adding the result to (24), we get

$$
\left\|A_{1}\right\|_{p}^{2}+\left\|A_{2}\right\|_{p}^{2} \leq \frac{2^{1-\frac{2}{p}}}{p}\left(\left\|A_{1}+A_{2}\right\|_{p}^{p}+\left\|A_{1}-A_{2}\right\|_{p}^{p}\right)^{\frac{2}{p}} .
$$

Since $\frac{1}{2} \leq \frac{2^{1-\frac{2}{p}}}{p}$, for $1 \leq p \leq 2$, (23) is sharper than (25). 
The space $C_{p}(n)$ is a Banach space with norm $\|\bar{A}\|_{p}=\left(\sum_{j=1}^{n}\left\|A_{j}\right\|_{p}^{p}\right)^{1 / p}$. Each operator $R$ in $B\left(H^{n}\right)$ defines a bounded operator on $C_{p}(n): \bar{A} \rightarrow R \bar{A}$, which we also denote by $R$. It follows from the second inequality of Theorem 1 that $\|R\|_{C_{p}(n)} \leq n^{\left|\frac{1}{p}-\frac{1}{2}\right|}\|R\|$. Each $P \in \mathcal{P}_{n}$ generates an isomorphism $\theta_{P}$ of $B(H)$ onto $B\left(H^{n}\right): \theta_{P}(R)=\left(R_{j k}\right)$, where $R_{j k}=P_{j} R P_{k}$. Set

$$
\|R\|_{p, n}=\sup \left\{\left\|\theta_{P}(R)\right\|_{C_{p}(n)}: P \in \mathcal{P}_{n}\right\} .
$$

The above argument yields

Corollary 8. $\|R\|_{p, n} \leq n^{\left|\frac{1}{p}-\frac{1}{2}\right|}\|R\|$ for $0<p<\infty$.

Proof of Theorem 5. For $\bar{A}=\left(A_{j}\right) \in C_{p}(n)$, let $A_{j}=X_{j}+i Y_{j}$, where $X_{j}, Y_{j}$ are selfadjoint operators. Set $T_{2 j}=A_{j}$ and $T_{2 j-1}=A_{j}^{*}$. Then $\bar{T} \in C_{p}(2 n)$,

$\left\|T_{2 j-1}\right\|_{p}=\left\|T_{2 j}\right\|_{p}=\left\|A_{j}\right\|_{p}$ and $T_{2 j-1}^{*} T_{2 j-1}+T_{2 j}^{*} T_{2 j}=A_{j} A_{j}^{*}+A_{j}^{*} A_{j}=2\left(X_{j}^{2}+Y_{j}^{2}\right)$.

Taking this into account and using Lemma 6 for $\bar{T}$, we obtain the proof of $(i)$.

Now set $S_{2 j-1}=X_{j}$ and $S_{2 j}=Y_{j}$. Then $\bar{S} \in C_{p}(2 n)$. Consider the $2 n \times 2 n$ block-matrix operator $R=\left(R_{k m}\right)$ such that all $R_{k k}=\mathbf{1}, R_{2 j-12 j}=R_{2 j 2 j-1}=i \mathbf{1}$, for $j=1, \ldots, n$, and all the other entries $R_{k m}=0$. It can be considered as an $n \times n$ block-matrix diagonal operator with each diagonal $2 \times 2$ block $\left(\begin{array}{cc}\mathbf{1} & i \mathbf{1} \\ i \mathbf{1} & \mathbf{1}\end{array}\right)$. Then $\beta=\|R\|=\left\|R^{*} R\right\|^{1 / 2}=2^{1 / 2}$ and $\alpha=\left\|R^{-1}\right\|=2^{-1 / 2}$. Set $\bar{B}=R \bar{S}$. Then $B_{2 j-1}=X_{j}+i Y_{j}=A_{j}$ and $B_{2 j}=i X_{j}+Y_{j}=i A_{j}^{*}$. Replacing $\bar{A}$ by $\bar{S}$ and $n$ by $2 n$ in Theorem 1 we obtain the proof of $(i i)$.

\section{Proof of Theorem 2}

The proof is similar to the proof of Theorem 4 of [BK3] and should be read together with it. It is based on a refinement of the lemma in Section 5 of [BK3]. Note first that, for $A \in C_{p}$,

$$
|A|^{\lambda} \in C_{p / \lambda} \text { and }\left\||A|^{\lambda}\right\|_{p / \lambda}=\|A\|_{p}^{\lambda} \text {, for } \lambda>0 \text {. }
$$

Lemma 9. Let $1<p \leq 2$ and $\bar{A} \in C_{p}(n)$. For $R=\left(R_{j k}\right) \in B\left(H^{n}\right)$, set $\bar{B}=R \bar{A}$ and $r=\max \left\|R_{j k}\right\|$. Then, for $\bar{Y} \in C_{q}(n)$,

$$
\left|\operatorname{Tr} \sum_{j=1}^{n} Y_{j}^{*} B_{j}\right| \leq r^{1-\frac{2}{q}} \beta^{2 / q}\left(\sum_{k=1}^{n}\left\|A_{j}\right\|_{p}^{p}\right)^{\frac{1}{p}}\left(\sum_{j=1}^{n}\left\|Y_{j}\right\|_{q}^{p}\right)^{\frac{1}{p}} \text {, where } q=\frac{p}{p-1}
$$

Proof. Let $A_{j}=\left|A_{j}\right| U_{j}$ and $Y_{j}=\left|Y_{j}\right| V_{j}$ be the polar decompositions. For $z=$ $x+i y, \frac{1}{2} \leq x \leq 1$, set

$$
A_{j}(z)=\left|A_{j}\right|^{p z} U_{j} \text { and } Y_{j}(z)=\left\|Y_{j}\right\|_{q}^{p z-q(1-z)}\left|Y_{j}\right|^{q(1-z)} V_{j} .
$$

By (26), $A_{j}(z) \in C_{x^{-1}}$ and $Y_{j}(z) \in C_{(1-x)^{-1}}$,

$$
\begin{aligned}
\left\|A_{j}(z)\right\|_{x^{-1}} & =\left\|\left|A_{j}\right|^{p x}\right\|_{x^{-1}}=\left\|A_{j}\right\|_{p}^{p x} \\
\text { and }\left\|Y_{j}(z)\right\|_{(1-x)^{-1}} & =\left\|Y_{j}\right\|_{q}^{p x-q(1-x)}\left\|\left|Y_{j}\right|^{q(1-x)}\right\|_{(1-x)^{-1}}=\left\|Y_{j}\right\|_{q}^{p x} .
\end{aligned}
$$


Set $\overline{B(z)}=R \overline{A(z)}$ and

$$
f(z)=\operatorname{Tr}(\overline{B(z)}, \overline{Y(z)})=\operatorname{Tr} \sum_{j=1}^{n} Y_{j}(z)^{*} B_{j}(z) .
$$

Let $z_{1}=1+i y$. Then all $Y_{j}\left(z_{1}\right) \in B(H)$ and $A_{j}\left(z_{1}\right) \in C_{1}$, so $B_{j}\left(z_{1}\right) \in C_{1}$. Hence

$$
\begin{aligned}
\left|f\left(z_{1}\right)\right| & =\left|\operatorname{Tr} \sum_{j=1}^{n} Y_{j}\left(z_{1}\right)^{*} B_{j}\left(z_{1}\right)\right| \leq\left\|\sum_{j=1}^{n} Y_{j}\left(z_{1}\right)^{*} B_{j}\left(z_{1}\right)\right\|_{1} \leq \sum_{j=1}^{n}\left\|Y_{j}\left(z_{1}\right)\right\|\left\|B_{j}\left(z_{1}\right)\right\|_{1} \\
& \leq \sum_{j=1}^{n}\left\|Y_{j}\right\|_{q}^{p}\left(\sum_{k=1}^{n}\left\|R_{j k}\right\|\left\|A_{k}\right\|_{p}^{p}\right) \leq r\left(\sum_{j=1}^{n}\left\|Y_{j}\right\|_{q}^{p}\right)\left(\sum_{k=1}^{n}\left\|A_{k}\right\|_{p}^{p}\right)=M_{1} .
\end{aligned}
$$

For $z_{2}=\frac{1}{2}+i y$, all $B_{j}\left(z_{2}\right) \in C_{2}$ and $Y_{j}\left(z_{2}\right) \in C_{2}$. By Theorem 1 ,

$$
\begin{aligned}
\left|f\left(z_{2}\right)\right| & \leq\left\|\sum_{j=1}^{n} Y_{j}\left(z_{2}\right)^{*} B_{j}\left(z_{2}\right)\right\|_{1} \leq \sum_{j=1}^{n}\left\|Y_{j}\left(z_{2}\right)\right\|_{2}\left\|B_{j}\left(z_{2}\right)\right\|_{2} \\
& \leq\left(\sum_{j=1}^{n}\left\|Y_{j}\left(z_{2}\right)\right\|_{2}^{2}\right)^{\frac{1}{2}}\left(\sum_{j=1}^{n}\left\|B_{j}\left(z_{2}\right)\right\|_{2}^{2}\right)^{\frac{1}{2}} \\
& \leq\left(\sum_{j=1}^{n}\left\|Y_{j}\right\|_{q}^{p}\right)^{\frac{1}{2}} \beta\left(\sum_{j=1}^{n}\left\|A_{j}\left(z_{2}\right)\right\|_{2}^{2}\right)^{\frac{1}{2}} \\
& \leq \beta\left(\sum_{j=1}^{n}\left\|Y_{j}\right\|_{q}^{p}\right)^{\frac{1}{2}}\left(\sum_{k=1}^{n}\left\|A_{j}\right\|_{p}^{p}\right)^{\frac{1}{2}}=M_{2} .
\end{aligned}
$$

Since $\frac{1}{2} \leq \frac{1}{p} \leq 1$, we have from the three-line theorem that

$$
\left|f\left(\frac{1}{p}\right)\right|=\left|\operatorname{Tr} \sum_{j=1}^{n} Y_{j}^{*} B_{j}\right| \leq M_{1}^{2\left(\frac{1}{p}-\frac{1}{2}\right)} M_{2}^{2\left(1-\frac{1}{p}\right)},
$$

which completes the proof of the lemma.

Proceed now as in BK3. Consider the polar decomposition $B_{j}=W_{j}\left|B_{j}\right|$ and set $Y_{j}=\left\|B_{j}\right\|_{p}^{q-p} W_{j}\left|B_{j}\right|^{p-1}$. By (26),$Y_{j} \in C_{q}$, where $q=\frac{p}{p-1}$,

$$
\left\|Y_{j}\right\|_{q}=\left\|B_{j}\right\|_{p}^{q-p}\left\|\left|B_{j}\right|^{p-1}\right\|_{q}=\left\|B_{j}\right\|_{p}^{q-1}
$$

and

$$
\operatorname{Tr}\left(Y_{j}^{*} B_{j}\right)=\left\|B_{j}\right\|_{p}^{q-p} \operatorname{Tr}\left(\left|B_{j}\right|^{p}\right)=\left\|B_{j}\right\|_{p}^{q} .
$$

Using Lemma 9, we complete the proof of Theorem 2 for $1<p \leq 2$.

To prove the theorem for $2 \leq p<\infty$, we use a duality argument. For $\bar{A} \in C_{p}(n)$, the $n \times n$ block-diagonal operator $A=\left(A_{j k}\right)$ with $A_{j j}=A_{j}$ belongs to the Schatten ideal $C_{p}\left(H^{n}\right)$ of operators on $H^{n}$ and defines a bounded functional $F_{A}$ on $C_{q}\left(H^{n}\right)$ 
by the formula

$$
\begin{aligned}
F_{A}(X)=\operatorname{Tr}\left(A^{*} X\right)=\operatorname{Tr}\left(\sum_{j=1}^{n} A_{j}^{*} X_{j j}\right) & =\operatorname{Tr}((\bar{X}, \bar{A})) \\
& \text { with }\left\|F_{A}\right\|=\|A\|_{p}=\left(\sum_{j=1}^{n}\left\|A_{j}\right\|_{p}^{p}\right)^{1 / p},
\end{aligned}
$$

where $\bar{X}=\left(X_{j j}\right) \in C_{q}(n)$. Let $\widehat{X}$ be the block-diagonal operator with diagonal blocks $X_{j j}$. Then $\|\widehat{X}\|_{q}=\left(\sum_{j=1}^{n}\left\|X_{j j}\right\|_{q}^{q}\right)^{1 / q}$ and (see [GK] $)\|\widehat{X}\|_{q} \leq\|X\|_{q}$. Hence

$$
\begin{aligned}
\|A\|_{p}=\sup \frac{\left|F_{A}(X)\right|}{\|X\|_{q}} \leq \sup \frac{|\operatorname{Tr}((\bar{X}, \bar{A}))|}{\|\widehat{X}\|_{q}} & =\sup \frac{\left|\operatorname{Tr}\left(\left(\bar{X}, R^{-1} \bar{B}\right)\right)\right|}{\|\widehat{X}\|_{q}} \\
& =\sup \frac{\left|\operatorname{Tr}\left(\left(\left(R^{-1}\right)^{*} \bar{X}, \bar{B}\right)\right)\right|}{\|\widehat{X}\|_{q}} .
\end{aligned}
$$

Let $\rho=\max \left\|\left(R^{-1}\right)_{i j}\right\|$ and $\alpha=\left\|R^{-1}\right\|$. Since $1 \leq q \leq 2$, exchanging $p$ and $q$ in Lemma 9, we have

$$
\begin{aligned}
\left|\operatorname{Tr}\left(\left(\left(R^{-1}\right)^{*} \bar{X}, \bar{B}\right)\right)\right| & \leq \rho^{1-\frac{2}{p}} \alpha^{\frac{2}{p}}\left(\sum_{j=1}^{n}\left\|X_{j j}\right\|_{q}^{q}\right)^{\frac{1}{q}}\left(\sum_{j=1}^{n}\left\|B_{j}\right\|_{p}^{q}\right)^{\frac{1}{q}} \\
& =\rho^{1-\frac{2}{p}} \alpha^{\frac{2}{p}}\|\widehat{X}\|_{q}\left(\sum_{j=1}^{n}\left\|B_{j}\right\|_{p}^{q}\right)^{\frac{1}{q}} .
\end{aligned}
$$

Combining the above inequalities, we complete the proof of the theorem for $2 \leq$ $p<\infty$.

\section{Generalization of Hirzallah-Kittaneh inequalities: Theorem 3}

Let $A$ be a positive operator. If $g$ is a convex function on $[0, \infty$ ), then (see Lemma 1 of $[\mathrm{HK}]$ )

$$
g(\langle A x, x\rangle) \leq\langle g(A) x, x\rangle, \text { for } x \in H \text { with }\|x\|=1 .
$$

If $g$ is concave, the inequality is reversed. A continuous function $g$ on $[0, \infty)$ is operator monotone if $0<A \leq B$ implies $g(A) \leq g(B)$ (see [B]).

To generalize Hirzallah-Kittaneh inequalities we need the following result, which gives us some useful inequalities for $(\bar{A}, \bar{A})$. Its proof essentially repeats the elegant proof of Theorem 1 in [HK] and uses Bhatia-Kittaneh's generalization (see [BK3]) of the Ando-Zhan theorem (see [AZ]).

Proposition 10. (i) Let $g$ be an increasing function on $[0, \infty)$ such that $g(0)=0$ and $\lim _{t \rightarrow \infty} g(t)=\infty$ and let its inverse function $g^{-1}$ be operator monotone. Then, for each s.n. ideal $J$ and all $\bar{A} \in J(n)$,

$$
\left\|\sum_{j} g\left(\frac{1}{n}\left|A_{j}\right|^{2}\right)\right\|_{J} \leq\left\|g\left(\frac{1}{n}(\bar{A}, \bar{A})\right)\right\|_{J} \leq \frac{1}{n}\left\|\sum_{j} g\left(\left|A_{j}\right|^{2}\right)\right\|_{J} .
$$


(ii) Let $g$ be a nonnegative operator monotone function on $[0, \infty)$. Then, for each s.n. ideal $J$ and each set $\bar{A}=\left\{A_{j}\right\}_{j=1}^{n}$ of operators in $B(H)$ such that $g(t(\bar{A}, \bar{A})) \in J$, for all $t>0$, the above inequalities are reversed.

Proof. As $g^{-1}$ is operator monotone, it is concave (see p.120 of [B]). Since $g$ is increasing, it implies that $g$ is convex. Let $\bar{A} \in J(n)$. Then, for each unit vector $x$ in $H$,

$$
\begin{aligned}
& \left\langle\sum_{j} g\left(\left|A_{j}\right|^{2}\right) x, x\right\rangle=\sum_{j}\left\langle g\left(\left|A_{j}\right|^{2}\right) x, x\right\rangle \stackrel{\sqrt{27}}{\geq} \sum_{j} g\left(\left\langle\left|A_{j}\right|^{2} x, x\right\rangle\right) \\
& \geq n g\left(\frac{1}{n} \sum_{j}\left\langle\left|A_{j}\right|^{2} x, x\right\rangle\right)=n g\left(\frac{1}{n}\langle(\bar{A}, \bar{A}) x, x\rangle\right) \text { (by convexity of } g, \text { cf. (18) ). }
\end{aligned}
$$

Set $B=\frac{1}{n}(\bar{A}, \bar{A})$. Then $\operatorname{Sp}(B) \subseteq[0, a]$ for some $a>0$. Since $g$ is convex and $g(0)=0$, we have $g(x)=x h(x)$ where $h$ is a bounded function on $(0, a]$. Hence $h(B) \in B(H)$, so $g\left(\frac{1}{n}(\bar{A}, \bar{A})\right)=g(B)=B h(B) \in J$.

As $g$ is an increasing function, setting $f(t)=t$ in (20), we obtain that

$$
\left\|\sum_{j} g\left(\left|A_{j}\right|^{2}\right)\right\|_{J} \geq n\left\|g\left(\frac{1}{n}(\bar{A}, \bar{A})\right)\right\|_{J}=n\left\|g\left(\frac{1}{n} \sum_{j}\left|A_{j}\right|^{2}\right)\right\|_{J} .
$$

Since $g^{-1}$ is operator monotone, it follows from Theorem $5(i i)$ of BK3] that

$$
\left\|g\left(\frac{1}{n} \sum_{j}\left|A_{j}\right|^{2}\right)\right\|_{J} \geq\left\|\sum_{j} g\left(\frac{1}{n}\left|A_{j}\right|^{2}\right)\right\|_{J}
$$

Combining the above inequalities, we complete the proof of part $(i)$.

If $g$ is an operator monotone function, it is concave. Using this (cf. (17)) and the reverse of (27), we obtain, similar to the above, that for each unit vector $x$ in $H$,

$$
\left\langle\sum_{j} g\left(\left|A_{j}\right|^{2}\right) x, x\right\rangle \leq n g\left(\frac{1}{n}\langle(\bar{A}, \bar{A}) x, x\rangle\right) .
$$

Since $g\left(\frac{1}{n}(\bar{A}, \bar{A})\right) \in J$ and $g$ is an increasing function, we have from (20) that $\sum_{j} g\left(\left|A_{j}\right|^{2}\right) \in J$ and

$$
\left\|\sum_{j} g\left(\left|A_{j}\right|^{2}\right)\right\|_{J} \leq n\left\|g\left(\frac{1}{n}(\bar{A}, \bar{A})\right)\right\|_{J}=n\left\|g\left(\frac{1}{n} \sum_{j}\left|A_{j}\right|^{2}\right)\right\|_{J} .
$$

Since $g$ is operator monotone, it follows from Theorem $5(i)$ of [BK3] that

$$
\left\|g\left(\frac{1}{n} \sum_{j}\left|A_{j}\right|^{2}\right)\right\|_{J} \leq\left\|\sum_{j} g\left(\frac{1}{n}\left|A_{j}\right|^{2}\right)\right\|_{J} .
$$

Combining the above inequalities, we complete the proof. 
Corollary 11. Let $R$ be an invertible $n \times n$ block-matrix operator, $\alpha=\left\|R^{-1}\right\|$, $\beta=\|R\|$. Let $J$ be an s. n. ideal and $\bar{B}=R \bar{A}$ for $\bar{A} \in J(n)$. If a function $g$ satisfies the conditions of Proposition $10(i)$, then

$$
n\left\|\sum_{j} g\left(\frac{\alpha^{-2}\left|A_{j}\right|^{2}}{n}\right)\right\|_{J} \leq\left\|\sum_{j} g\left(\left|B_{j}\right|^{2}\right)\right\|_{J} \leq \frac{1}{n}\left\|\sum_{j} g\left(\beta^{2} n\left|A_{j}\right|^{2}\right)\right\|_{J} .
$$

If $g$ satisfies the conditions of Proposition 10(ii), then

$$
n\left\|\sum_{j} g\left(\frac{\beta^{2}\left|A_{j}\right|^{2}}{n}\right)\right\|_{J} \geq\left\|\sum_{j} g\left(\left|B_{j}\right|^{2}\right)\right\|_{J} \geq \frac{1}{n}\left\|\sum_{j} g\left(\alpha^{-2} n\left|A_{j}\right|^{2}\right)\right\|_{J} .
$$

Proof. As in (22), we have $(\bar{A}, \bar{A}) \leq(\alpha \bar{B}, \alpha \bar{B})$ and $(\bar{B}, \bar{B}) \leq(\beta \bar{A}, \beta \bar{A})$. Let $g$ satisfy the conditions of Proposition $10(i)$. Then

$$
\begin{aligned}
n\left\|\sum_{j} g\left(\frac{\left|A_{j}\right|^{2}}{n}\right)\right\|_{J} \leq n\left\|g\left(\frac{(\bar{A}, \bar{A})}{n}\right)\right\|_{J} & \stackrel{200}{\leq} n\left\|g\left(\frac{(\alpha \bar{B}, \alpha \bar{B})}{n}\right)\right\|_{J} \\
& \leq\left\|\sum_{j} g\left(\left|\alpha B_{j}\right|^{2}\right)\right\|_{J} .
\end{aligned}
$$

Similarly,

$$
n\left\|\sum_{j} g\left(\frac{1}{n}\left|B_{j}\right|^{2}\right)\right\|_{J} \leq\left\|\sum_{j} g\left(\left|\beta A_{j}\right|^{2}\right)\right\|_{J} .
$$

Combining these inequalities yields the proof of (28).

As $(\bar{B}, \bar{B}) \leq(\beta \bar{A}, \beta \bar{A})$, it follows from (20) that if $g(t(\bar{A}, \bar{A})) \in J$, for all $t>0$, then $g(t(\bar{B}, \bar{B})) \in J$. Taking this into account, we obtain (29) in the same way as (28).

Proof of Theorem 3. For $0<k<1$, the function $g(t)=t^{k}$ is operator monotone; for $k \geq 1$, the inverse of $g$ is operator monotone (see [B, p. 115]). Setting $p=2 k$, we obtain Theorem 3 from (28) and (29).

\section{REFERENCES}

[AZ] T. Ando and X. Zhan, Norm inequalities related to operator monotone functions, Math. Ann., 315(1999), 771-780. MR 1727183 (2000m:47008)

[BCL] K. Ball, E.A. Carlen and E.H. Lieb, Sharp uniform convexity and smoothness inequalities for trace norms, Invent. Math., 115(1994), 463-482. MR1262940 (95e:47027)

[B] R. Bhatia, Matrix Analysis, Springer, New York, 1997. MR1477662 (98i:15003)

$[\mathrm{BH}]$ R. Bhatia and J.A.R. Holbrook, On the Clarkson-McCarthy inequalities, Math. Ann., 281(1988), 7-12. MR0944598 (89m:47005)

[BK1] R. Bhatia and F. Kittaneh, Norm inequalities for partitioned operators and applications, Math. Ann. 287(1990), 719-726. MR1066826 (91f:47010)

[BK2] R. Bhatia and F. Kittaneh, Cartesian decompositions and Schatten norms, Linear Algebra and Appl., 318(2000), 109-116. MR1787227(2002k:47021)

[BK3] R. Bhatia and F. Kittaneh, Clarkson inequalities with several operators, Bull. London Math. Soc., 36(2004), 820-832. MR2083758 (2005m:47015)

[GK] I. C. Gohberg and M.G. Krein, Introduction to the theory of linear non-selfadjoint operators in Hilbert space, Nauka, Moscow, 1965. MR0220070 (36:3137) 
[HK] O. Hirzallah and F. Kittaneh, Non-commutative Clarkson inequalities for unitarily invariant norms, Pacific J. Math., 202(2002), 363-369. MR.1887770 (2003a:47020)

[M] C.A. McCarthy, $c_{p}$, Isr. J. Math., 5(1967), 249-271. MR0225140(37:735)

[S] B. Simon, Trace ideals and their applications, Cambridge University Press, 1979. MR.0541149 (80k:47048)

Department of Computing, Communications Technology and Mathematics, London Metropolitan University, 166-220 Holloway Road, London N7 8DB, Great Britain

E-mail address: e.kissin@londonmet.ac.uk 